\title{
$\underline{\text { Two Decades of Mexican Particle Physics at Fermilab }}$
}

\author{
Roy Rubinstein \\ Fermilab
}

This report is a view from Fermilab of Mexican particle physics at the Laboratory since about 1980; it is not intended to be a history of Mexican particle physics: that topic is outside the expertise of the writer. The period 1980 to the present coincides with the growth of Mexican experimental particle physics from essentially no activity to its current state where Mexican groups take part in experiments at several of the world's major laboratories.

Soon after becoming Fermilab director in 1979, Leon Lederman initiated a program to encourage experimental physics, especially experimental particle physics, in Latin America. At the time, Mexico had significant theoretical particle physics activity, but none in experiment. Following a visit by Lederman to UNAM in 1981, a conference "Panamerican Symposium on Particle Physics and Technology" was held in January 1982 at Cocoyoc, Mexico, with about 50 attendees from Europe, North America, and Latin America; these included Lederman, M. Moshinsky, J. Flores, S. Glashow, J. Bjorken, and G. Charpak. Among the conference outcomes were four subsequent similar symposia over the next decade, and a formal Fermilab program to aid Latin American physics (particularly particle physics); it also influenced a decision by Mexican physicist Clicerio Avilez to switch from theoretical to experimental particle physics.

The first physics collaboration between Fermilab and Mexico was in particle theory. Post-docs Rodrigo Huerta and Jose Luis Lucio spent 1-2 years at Fermilab starting in 1981, and other theorists (including Augusto Garcia, Arnulfo Zepeda, Matias Moreno and Miguel Angel Perez) also spent time at the Laboratory in the 1980s.

There were two major inputs to Mexican experimental particle physics at Fermilab, and these were provided by Clicerio Avilez, and by a PhD program in experimental particle physics started in the early 1980s at CINVESTAV.

Clicerio Avilez (1945-91) was a UNAM theoretical particle physicist, who made the decision to switch to experiment in the early 1980s. He spent a year at Fermilab in 1984/85, working on experimental particle physics and also on accelerator physics (he was interested in medical accelerators for Mexico). Avilez joined E-766 (hyperon production) at Brookhaven National Laboratory, and was an important member of Fermilab E-690 (strange/charm production). In 1987, he moved to the University of Guanajuato and started to build a group there to carry 
out experiments at the major laboratories of the world-the first such group in Mexico. By the end of the 1980s, he was a frequent visitor to Fermilab, and was also internationally known as a leader of Mexican particle physics; his untimely death was a significant blow to particle physics in Mexico.

The other major input to Mexican experimental particle physics was provided by CINVESTAV. This institute had a PhD program in theoretical particle physics, and in the early 1980s it decided to start one in experimental particle physics; however, at the time CINVESTAV had nobody on the faculty working in this field. Three CINVESTAV students (Hector Mendez, Antonio Morelos and Gerardo Moreno) joined the program; for their thesis research work, they came to Fermilab for several years, each one joining an experiment here, and each having a Fermilab staff member as thesis advisor. Around 1990, all three students were awarded their CINVESTAV PhDs, and all three have continued since then to be active in the field. The CINVESTAV PhD program in experimental particle physics became established, and now the institute has experimentalists on the faculty to supervise the theses of its experimental students, and is active in international particle physics experiments.

In the 1990s, there was a significant expansion in experimental particle physics in Mexico. New groups were formed, and funding became available from Mexican sources. Several Fermilab fixed target experiments ran during the decade with collaborators from Mexican institutions; they were E-690 (Guanajuato), E-781 (San Luis Potosi), E-791 (CINVESTAV, Puebla), E-831 (CINVESTAV, Puebla), and E-871 (Guanajuato); all experiments are still analyzing data and producing physics results. In addition, a CINVESTAV group was, and is, a member of the D0 Tevatron collider experiment. Many published papers from Fermilab experiments now have Mexican authors and institutions listed.

In the 2000s up to the present time, CINVESTAV continues to be a member of D0, and San Luis Potosi is a member of the CKM collaboration, which will study the important rare decay $\mathrm{K}+\rightarrow \mathrm{pi}+$ nu nubar. There are currently experimental particle physics activities at CINVESTAV, CINVESTAV-Merida, Guanajuato, Morelia, Puebla, and San Luis Potosi, and Mexican groups continue to play an important part in the Fermilab research program. An important change in Mexican experimental particle physics over the past two decades is that whereas in the 1980s it was almost entirely centered at Fermilab, there are now Mexican user activities at DESY, CERN and on the Auger project.

In addition to the activities of Mexican physicists at Fermilab discussed above, many Fermilab staff members have travelled to Mexico over the past twenty years for such activities as conferences, sabbaticals and collaboration meetings. 
Mexican experimental particle physics essentially did not exist two decades ago. Now Mexican groups are important players in experiments around the world. This is a very impressive growth. Fermilab is pleased to have played a small part in this, but many people in Mexico worked very hard to reach this stage, and among them are the two people being honored by the Mexican Physical Society at this meeting, Augusto Garcia and Arnulfo Zepeda.

Presented at X Mexican School of Particles and Fields, Playa del Carmen, Mexico, 30 October 2002. 